\title{
Simulated Electrocortical Activity at Microscopic, Mesoscopic, and Global Scales
}

\author{
JJ Wright*,1,2,3,6, CJ Rennie ${ }^{1,4,5}$, GJ Lees ${ }^{2}$, PA Robinson ${ }^{1,4}$, PD Bourke ${ }^{3}$, CL Chapman ${ }^{3}$, E Gordon ${ }^{1,7}$ and DL \\ Rowe ${ }^{1,4}$ \\ 'Brain Dynamics Centre, Westmead Hospital and University of Sydney, Westmead, Australia; ${ }^{2}$ Department of Psychiatry, University of Auckland \\ School of Medicine, Auckland, New Zealand; ${ }^{3}$ Mental Health Research Institute, Parkville, Victoria, Australia; ${ }^{4}$ Theoretical Physics Group, School of \\ Physics, University of Sydney, Australia; ${ }^{5}$ Department of Medical Physics, Westmead Hospital, Westmead, Australia; ${ }^{6}$ Liggins Institute, University \\ of Auckland, Auckland, New Zealand; 'Department of Psychological Medicine, Westmead Hospital and University of Sydney, Westmead, \\ Australia
}

\begin{abstract}
Simulation of electrocortical activity requires (a) determination of the most crucial features to be modelled, (b) specification of state equations with parameters that can be determined against independent measurements, and (c) explanation of electrical events in the brain at several scales. We report our attempts to address these problems, and show that mutually consistent explanations, and simulation of experimental data can be achieved for cortical gamma activity, synchronous oscillation, and the main features of the EEG power spectrum including the cerebral rhythms and evoked potentials. These simulations include consideration of dendritic and synaptic dynamics, AMPA, NMDA, and GABA receptors, and intracortical and cortical/subcortical interactions. We speculate on the way in which Hebbian learning and intrinsic reinforcement processes might complement the brain dynamics thus explained, to produce elementary cognitive operations.

Neuropsychopharmacology (2003) 28, S80-S93. doi: I0.1038/sj.npp. I 300138
\end{abstract}

Keywords: gamma rhythm; cortical synchrony; evoked potentials; NMDA; AMPA; GABA

\section{INTRODUCTION}

Neuropsychopharmacology is a discipline attempting the unification of events at different scales in the brain. This is part of the larger task of relating brain events to conscious experience and learning processes of normal people and people suffering from mental disorders. The problems involved in this unification are legion, as is well known to readers of this journal. It is commonly found that different types of events in the brain are difficult to relate to each other, except as purely empirical findings. For example, it is seldom clear how findings made by PET and fMRI scanning are functionally related to most EEG measures. Nor how EEG measures might reflect specific changes in synaptic physiology. Nor how neurochemical effects can be functionally related to psychological events. These are all difficult questions, despite the huge growth in detailed knowledge about specific processes and components in the brain. No simple one-to-one ways exist, to relate these very complex phenomena, one to the others. Yet unifying principles must be sought, if the discipline is to

\footnotetext{
*Correspondence: Dr JJ Wright, Brain Dynamics Centre, Westmead Hospital, University of Sydney, Westmead, NSW 2145, Australia, Fax: +61 39387506 I, E-mail: jjw@mhri.edu.au

Received 0 I September 2002; revised 03 November 2002; accepted

03 December 2002
}

advance in a coherent fashion. One way of moving towards this unification is through the discipline of Brain Dynamics.

The aim of the subject of Brain Dynamics is to give a simplified, but sufficient, mathematical description of the operation of the brain, in terms of the brain's observable electrical activity (Freeman, 1975; Basar, 1976; Nunez, 1981, 1995).

Obtaining a 'simplified, but sufficient, mathematical description' can be broken down into a number of subtasks. These are:

(a) Abstraction from physiological data of the most important properties of the neurones of the brain, to be used in the mathematical model.

(b) Determination of the form of the state equations, and the values of parameters.

(c) Comparison of the equations' properties to observable aspects of brain function, at as many temporal and spatial scales as possible.

The observable aspects of brain function to be accounted for include the global EEG, the electrocorticogram and local field potentials, and the statistics of action potentials. If a wide match to experimental data can be achieved, then the model that has emerged should exhibit dynamic properties akin to a real brain, and it might then be asked what consequences these dynamic properties have for adaptive 
learning. Does the introduction of plausible learning rules then lead to an explanation of adaptive behavior?

Attempts to approach a theory of the brain along these lines stem from the work of McCulloch and Pitts (1943), and have led to extensive work on the properties of artificial neural networks (eg Amit, 1989). Lines of theoretical development more explicitly concerned with physiological dynamics, and especially the dynamics underlying EEG, flow from Wilson and Cowan (1973), Freeman (1975), Nunez (1981), Lopes da Silva (van Rotterdam et al, 1982), Haken (Haken et al, 1985), and others (eg Arbib et al, 1998). Work by Freeman (1975 and subsequently), and of Singer and Gray and others (eg Gray and Singer, 1989; Gray et al, 1989; Eckhorn et al, 1988) has been of particular importance in revealing the dynamics of the brain from an experimental perspective.

The work to be described from our own group has evolved from early attempts to define circumstances in which linear methods of analysis could be applied (Wright, 1990). Simple numerical simulations followed (Wright and Liley, 1996; Wright, 1997, 1999), leading to more advanced methods, including the development of wave equations (Robinson et al, 1997, 1998a, b, 2001). We have progressively introduced more refined physiological parameters, and descriptions of anatomical organization (Liley and Wright, 1994; Rennie et al, 1999, 2000, 2002), with the object of developing a single model to account for events in the brain at a number of different scales.

\section{OVERALL BRAIN ORGANIZATION}

Figure 1 highlights elementary features of gross brain organization. The cortical mantle is the terminating area for major sensory pathways, and the source of much of the signals organizing motor activity. The cortex never acts alone, but via continuous interaction with subcortical systems, notably the thalamus, the limbic system including hippocampus, the basal ganglia, etc (Alexander et al, 1990; Posner and Petersen, 1990). Any model complete to first approximation must consider interactions within the cortex, and also between cortex and subcortical structures, including events at microscopic, mesoscopic, and macroscopic (global or whole-brain) scales.

\section{CORTICAL DYNAMICS AT MICROSCOPIC SCALE}

Figure 2 shows the two elementary components of the cortex - excitatory (pyramidal) cells which make up about $90 \%$ of cortical cells, and which send axons to remote cortical locations, as well as interacting with near neighbors via intracortical axons - and inhibitory cells, which give only local intracortical axons. The excitatory cells utilize glutamate as a neurotransmitter, and act principally on fastacting AMPA receptors, and slower-acting NMDA receptors. The NMDA receptors are voltage dependent - that is, they influence the postsynaptic dendrites when the receiving cell is depolarized, and thereby emitting action potentials. The inhibitory cells use GABA as their neurotransmitter. These are the most important fast forms of neuronal interaction.

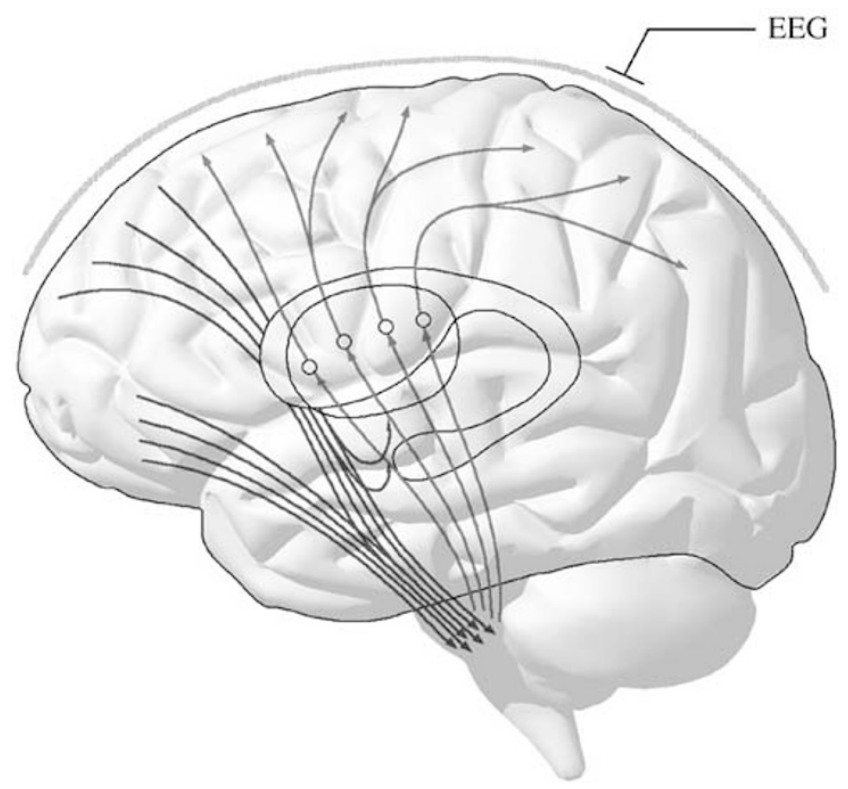

Figure I Overall brain organization and the EEG. The electroencephalogram arises from the cortical mantle. Cortical neurone cells interact locally with each other, and with many subcortical systems, each with complex delays and spatial dispersions.

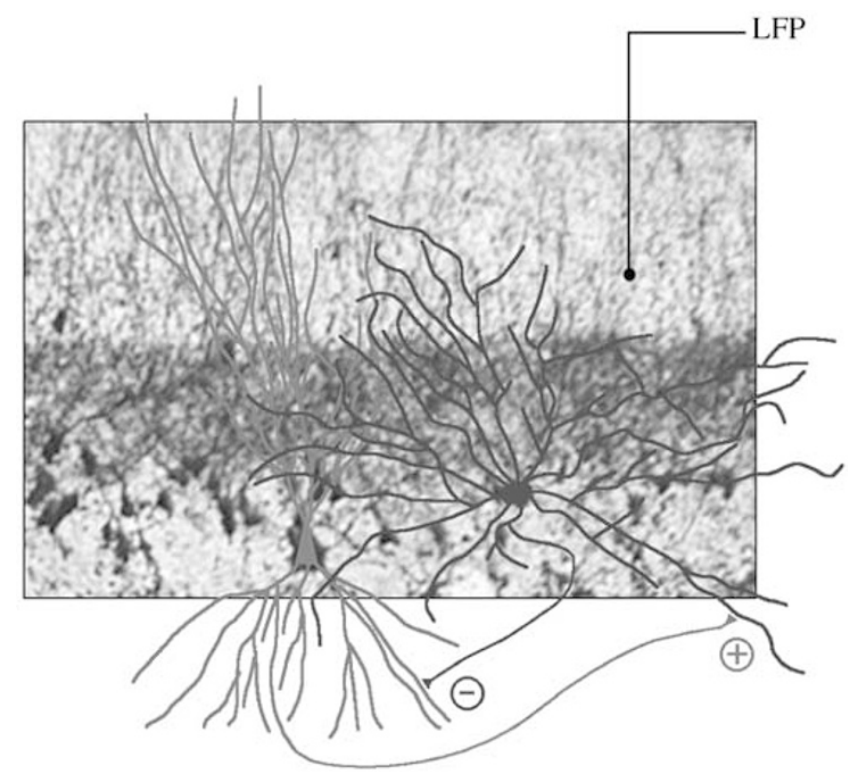

Figure 2 Neurones involved in intracortical interactions. Excitatory glutaminergic neurones (grey) interact locally with inhibitory gabaminergic neurones (black). Corticocortical connections arise from the excitatory cells and couple cortical locales together over long range.

A mathematical account of interactions in the cortex of these restricted types is given in the appendix, and the values we have obtained for all the parameters required in this mathematical model are given in Table 1 . The values of the parameters are taken largely from the findings of Braitenberg and Schuz (1991), Thomson et al (1996), and Thomson (1997), either directly, or from further calculations based on these (Liley and Wright, 1994; Rennie et al, 2000). Recent additions regarding synaptic physiology 
Table I State Variables and Parameters

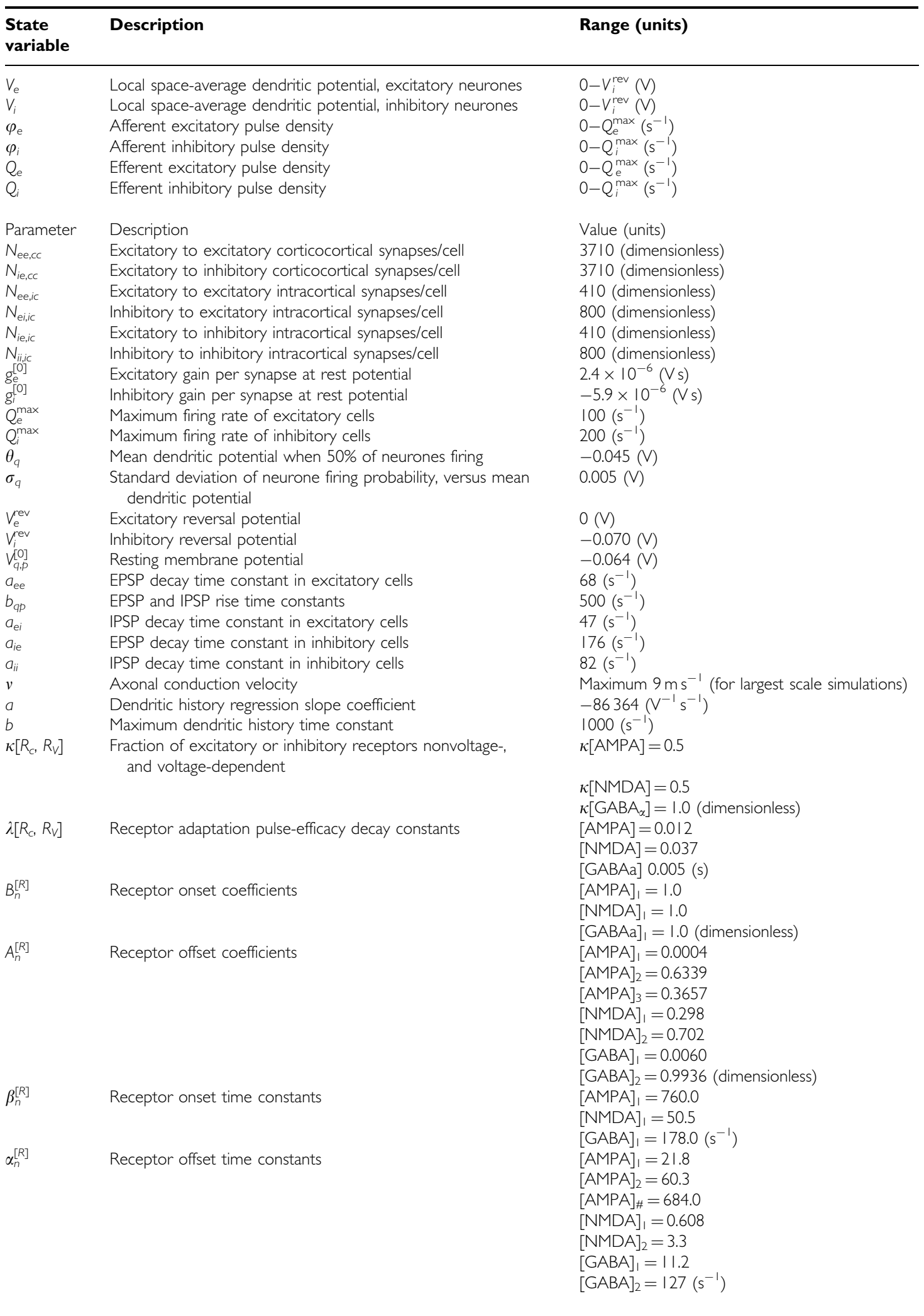

are derived from the findings of Tones and Westbrook (1996), Lester and Jahr (1992), Dominguez-Perrot et al (1996), Hausser and Roth (1997), and Partin et al (1996).

Our account treats the cortex as a continuum, rather than as a set of separate cells, although the properties of the continuum are closely related to the physiology of individual neurones. It is thus an account of population dynamics, rather than of cell-by-cell interactions, and is suitable for numerical solution of the events over a large extent of the cortical surface, as well as in small domains of 
macrocolumnar, or lesser, dimension. In addition to numerical solutions, the equations can be solved for some purposes in linear approximation, to yield wave equations and dispersion relations, etc (Robinson et al, 1997, 1998a, b; Rennie et al, 2000). Comparable treatments have been given in Jirsa and Haken (1996), Liley et al (1999, 2002), and Jirsa et al (2001).

The way the mathematics are related to the cortical anatomy and physiology is contained in three main equations, next described, with explanation of the minor terms and parameters left to the appendix and Table 1.

In the following formulae, subscript $p$ represents association with a presynaptic neurone, and subscript $q$ association with a postsynaptic neurone. Thus, $p$ or $q$ may each be replaced by either $e$ (excitatory) or $i$ (inhibitory), and subscript $q p$ indicates a property arising from the action of $p$ upon $q$.

The principal observable property, to which the EEG can be directly related, is the average dendritic membrane potential, or local field potential, arising from the pyramidal cells of the cerebral cortex. This is designated $V_{e}$, in accord with the above convention, when $q=e$.

The first equation describes the conversion of average dendritic membrane potentials within a small locale at a position $\mathbf{r}$, into the density of action potentials within the locale, as a sigmoidal function. Thus, $V_{q}(\mathbf{r}, t)$, the average membrane potential of excitatory or inhibitory cells becomes converted into $Q_{q}(\mathbf{r}, t)$, the instantaneous average firing rate of cells of type $q$, within the locale

$$
Q_{q}=Q_{q}^{\max } / 1+\mathrm{e}^{-\pi\left(V_{q}-\theta_{q}\right) / \sqrt{3} \sigma_{q}} .
$$

With the introduction of $\pi$ and $\sqrt{3}$, the parameters $\theta$ and $\sigma$ correspond, respectively, to the average membrane potential at which pulse density reaches $50 \%$ of maximum, and the standard deviation of pulse density as a function of average membrane potential. The probability that a randomly chosen cell within the continuum is emitting an action potential at a randomly chosen time, $t$, is given by $Q_{q} / Q_{q}^{\max }$.

Within the continuum formulation, $Q_{q}(\mathbf{r}, t)$ can be further treated as stochastic variables, by assuming that the Poisson distribution of action potentials emitted from individual neurones reflects random processes superimposed on the mass-action influences within the continuum. This random perturbation of instantaneous pulse density thus contributes one type of driving to the net cortical activity.

The second equation describes the way that action potentials reach the synapses at $\mathbf{r}$ from all the neurones elsewhere in the cortex. This synaptic flux, $\varphi_{p}$, arises from pulses, $Q_{p}$ which arose at many positions, $\mathbf{r}^{\prime}$, and were conveyed at the velocity of axonal conduction, $v_{p}$, to arrive later at $\mathbf{r}$ :

$$
\varphi_{p}=\int f\left(\left|\mathbf{r}-\mathbf{r}^{\prime}\right|, \mathbf{r}_{p}\right) Q_{p}\left(\mathbf{r}^{\prime}, t-\left|\mathbf{r}-\mathbf{r}^{\prime}\right| / v_{p}\right) \mathrm{d}^{3} \mathbf{r}^{\prime} .
$$

The final equation is more complicated, and describes the dynamics of the synapses, and the dendritic membranes, in converting the synaptic flux into the average dendritic potential, which then gives rise to further action potentials. This is

$$
V_{q}=V_{q}^{[0]}+\sum_{p} N_{q p} H_{q p}^{[D]} \otimes\left(s_{q p} \Gamma_{p} \varphi_{p}\right)
$$

including the densities of synapses of different types $\left(N_{q p}\right)$, the time characteristics of EPSP and IPSP, $\left(H_{q p}^{[D]}\right)$, the effects of reversal potentials and backpropagation of action potentials into the dendritic tree $\left(s_{q p}\right)$, and the modulation of AMPA, GABA, and NMDA receptor actions $\left(\Gamma_{p}\right)$. The last term, $\Gamma_{p}$, has parameters derived from recent models of the kinetics of channel opening and closing in receptors, consequent to varying equilibria among various tertiary molecular configurations of the receptor-channel complex (Lester and Jahr, 1992; Dominguez-Perrot et al, 1996; Hausser and Roth, 1997; Partin et al, 1996). The derivation of these receptor parameters will be reported elsewhere.

When compared to related and ancestral continuum models, the novel features of the present treatment are contained in the terms $\Gamma_{p}$ and $s_{q p}$.

The features describing the adaptations of AMPA, NMDA, and GABA receptors confer two properties. Firstly, because of the relatively rapid decrease in efficacy of the excitatory receptors compared to that of the inhibitory receptors as both types of synaptic flux increase, there is a tendency to stabilize locally the rate of generation of action potentials. Secondly, the voltage-dependent operation of NMDA receptors confers a capacity for the transient amplification of fields of synchronous oscillation, as will be shown below.

The features encapsulated in $s_{q p}$ have consequences for the frequency spectrum of local cortical activity. They confer a switch-like property, such that for relatively low pulse densities the 1/f background spectrum typical of EEG is the predominant feature. At a critical point as firing rate increases, a switch to oscillation in the gamma band occurs, of greater local amplitude than the $1 / f$ background. This switch depends crucially on a convolution, $H_{q}^{[M]} \otimes V_{q}$, contained in $s_{q p}$ (see appendix). Physically, $H_{q}{ }^{[M]}$ acts as a sharply adjusted filtering process conferring the switching effect, which is attributed to the impact of backpropagation of action potentials into the proximal dendritic tree (Stuart and Sakmann, 1994).

The combination of these properties enables simulation of a two-dimensional wave medium, which can be compared to experimental data, as is shown in Figures 3 and 4.

(a) At low levels of nonspecific cortical activation, when stimulated by weak afferent volleys (approximated as a white noise) to any part of the cortical surface, or in response to the intrinsic perturbations of $Q_{q}(\mathbf{r}, t)$ alone, wave motion propagates outward from the site of stimulation. The wave motion propagates at realistic speed for electrocortical activity and has a ' $1 / f$ ' frequency content, identical to that seen in background EEG activity. At very low levels of cortical activation, a small peak of activity is also seen in the theta range, but this cannot account fully for the power seen in the theta band in matched experimental data (Figure 3). The failure to account fully for the theta activity is one example of why the cortical simulation needs to be supplemented with consideration of cortical/subcortical interactions - see further below. 

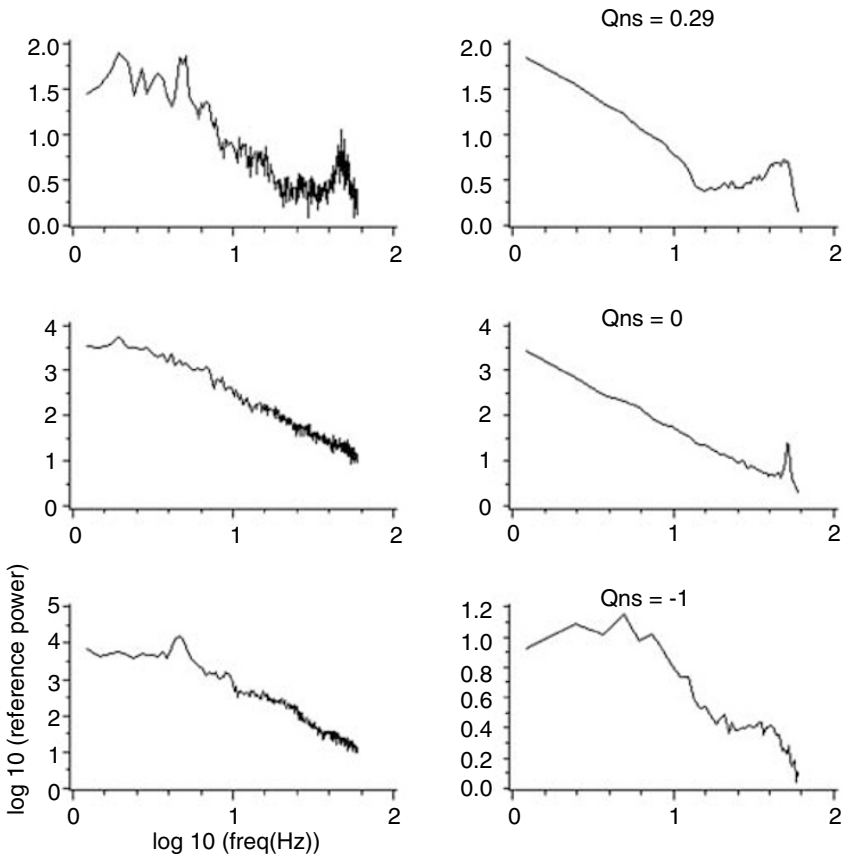

Figure 3 Log/log power spectra from real and simulated electrocorticogram. Experimental data (left-hand graphs) from Professor Walter Freeman's laboratory show typical samples of spectral content from neocortical sites in a variety of states of cortical activation. Simulated data (right-hand graphs) show, from top to bottom, performance of the cortical simulation at high, medium, and low levels of cortical activation. (Qns is a measure of cortical activation.) Note the model failure at the lowest level of cortical activation (see text for discussion)
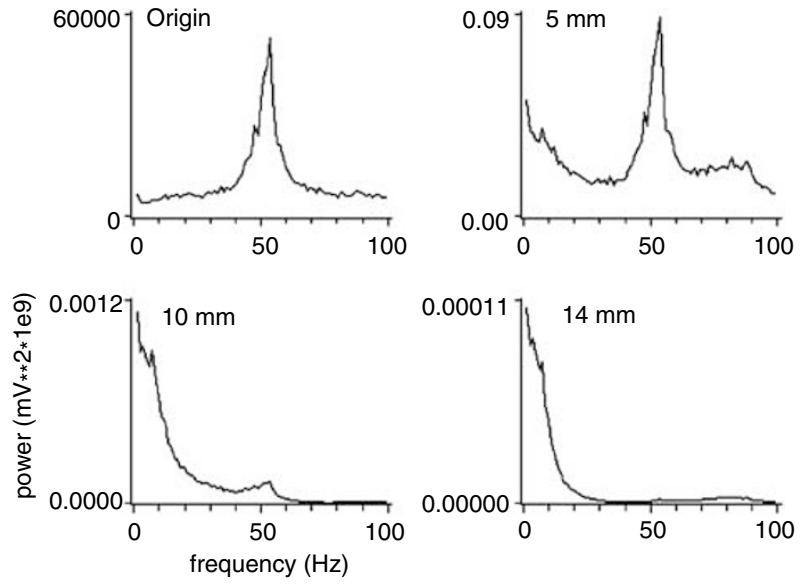

Figure 4 Spontaneous gamma activity interacting with the surrounding cortical field. Top left graph shows the power spectrum of electrocortical activity associated with gamma-band oscillation at a site on the cortical surface stimulated only by a DC input. Subsequent graphs show how the spectrum of activity is transformed during the propagation of waves through the quiescent surrounding cortex, to give rise to the I/f cortical background activity.

(b) At slightly higher levels of nonspecific cortical activation, broadband activity in the gamma range appears against the $1 / f$ background, and can be matched to experiments (Figure 3). (c) At still higher levels of nonspecific cortical activation, with or without introduction of specific sensory input, oscillation appears in the simulated cortical field, and this has close similarity to high levels of gamma or ' $40 \mathrm{~Hz}$ ' observed in many cortical experiments. Distant from the site of this autonomous oscillation, it is found that the gamma activity has acted as a driving source for the background $1 / f$ activity, by contributing a further increase in the amplitude of random fluctuations of pulse density. (Figure 4). At the site of the autonomous gamma oscillation, the signal amplitude is very high compared to the background $1 / f$ activity at remote sites, and is closely correlated with local pulse activity. Pulse densities are comparable to those observed physiologically (Steriade $e t$ $a l, 2001)$. At shorter distances of transmission, or with high coupling strength between locales, the direct transmission of activity in the gamma band is apparent.

Thus, depending upon local input conditions, transitions can take place between a condition of autonomous oscillation in the gamma band, and a dissipative, pointattractor condition, in which the cortex is a passive medium of wave transmission. Transition between these states occurs sharply, depending on a very small change in local cell firing rate, and near this transition, both the $1 / f$ background activity and the gamma activity have a stability factor close to zero. This capacity for sharp transition of state, analogous to a thermodynamic change of phase, bears comparison to the 'edge of chaos' type of dynamics described by Langton (1986, 1990), without necessarily implying that the cellular dynamics are chaotic in either state. The occurrence of fluctuating changes of state at a critical level of activation accords with physiological observations (Freeman and Rogers, 2002; Phillips and Pflieger, 1999). There are, in turn, possible implications for information processing, storage and recall from memory (Kay and Phillips, 1997; Phillips and Singer, 1997; Wright, 1997b; Arhem and Liljenstrom, 2001; Liljenstrom, 2002). Also, patches of active cortex can enter into synchronous oscillation, as is next described.

\section{CORTICAL INTERACTIONS AT MESOSCOPIC SCALE}

At a scale from fractions of a millimeter to many centimeters of cortex, patches of active cells have been experimentally observed to enter into synchronous oscillation - that is, cross-correlations of pulse density, or of mean local field potential at the separated loci are maximal at zero lag. Synchronous oscillation has been widely, although controversially, considered to act as a substrate for association processes in the cortex. (eg Eckhorn et al, 1988; Singer, 1994; Singer and Gray, 1995; Stryker, 1989; Bressler et al, 1993; Livingstone, 1996; Miltner et al, 1999; Neuenschwander and Singer, 1996; Palm and Wennekers, 1997; Steriade et al, 1996; Gray and Singer, 1989; Gray et al, 1989).

Results very similar to classic findings of synchronous oscillation readily appear when suitable stimuli are introduced into the simulated cortex, as is shown in Figure 5 . This property was first observed in numerical simulations (Traub et al, 1996; Wright, 1997a; Wright et al, 2000), and then explained analytically (Robinson et al, 1998a; Chap- 


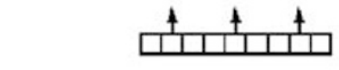

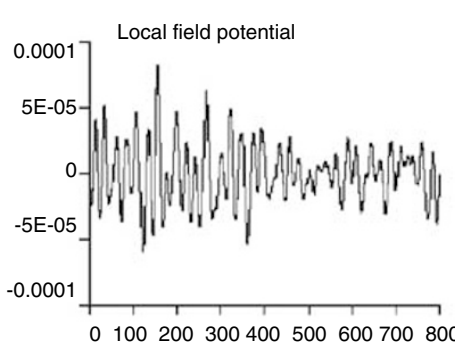

$\mathrm{ms}$
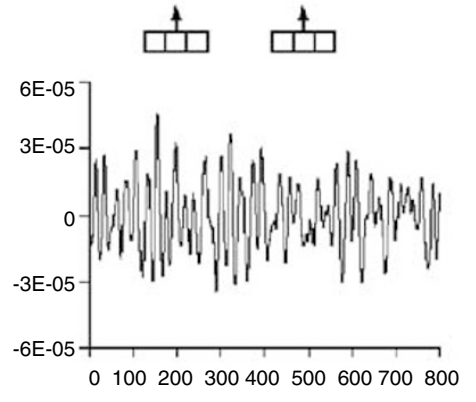

$\mathrm{ms}$
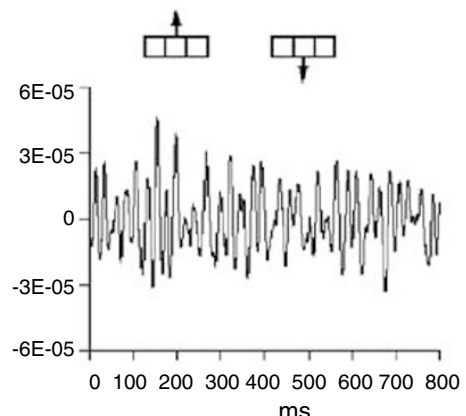

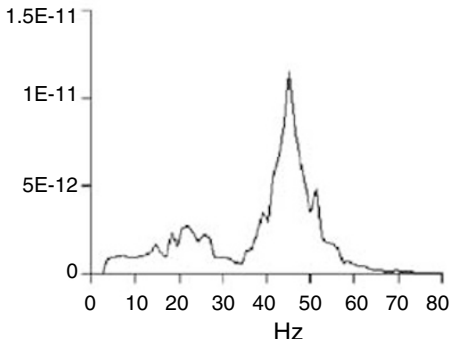

Pulse cross correlation
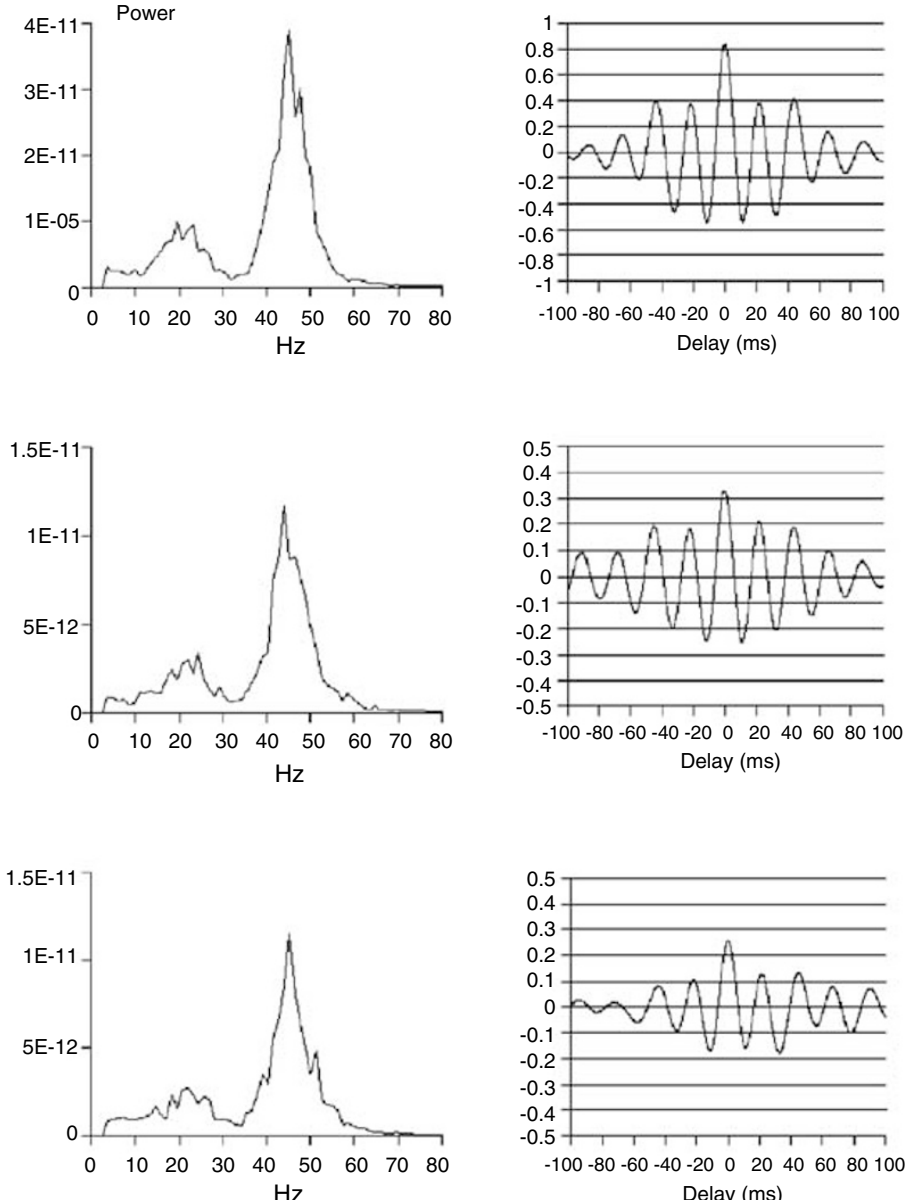

Figure 5 Simulation of synchronous oscillation induced by moving bars. Local field potential time series, power spectral content, and cross-correlations of two sites in the cortical receptive field, stimulated by combinations of moving bars in the visual field. Delivery of the visual stimuli to the visual cortex was simulated by the delivery of moving bars of zero-mean white noise to the simulated cortical surface. Input signals within a bar are spatially coherent, while the signals input to separate bars are uncorrelated. (In these simulations, the stimulus threshold for conversion to spontaneous oscillation in the gamma range was not exceeded.).

man et al, 2002). Cross-correlation at zero lag can be shown to occur at all frequencies, but, as is the case in physiological experiments, large oscillations in the gamma band, at about $40 \mathrm{~Hz}$, are associated with the largest amplitudes.

If two points on the simulated cortical surface are driven by separate, uncorrelated, inputs (or in response to uncorrelated autonomous local cortical activity at two separated points) then, within a few milliseconds crosscorrelated activity, maximal at zero lag, appears in the neighborhood of both active sites. For this effect to occur axonal delay must be small compared to the rise and fall time of the dendritic response, and the speed of onset of synchrony depends mainly on the axonal delay.

Figure 6 sums up the mechanism. The results shown in the top two rows of the figure are derived from a simulated field of electrocortical activity, which is not shown explicitly. The relevant state-variable in the simulated field was $V_{e}(\mathbf{r}, t)$, the mean dendritic potential of excitatory cells, and the field activity was generated by delivering two independent and uncorrelated time series of white noise to two points on the simulated cortical field. Wave activity radiated outward from both sites of input. The relevance for the generation of synchronous oscillation in the brain lies in the way these two independent fields of travelling waves can be shown to interact.

The top frames of Figure 6 show how a large field of zerolag synchrony surrounds both sites of uncorrelated input. The middle frames show eigenmode decomposition of the same field of wave activity, which breaks the field of activity into components of spatial activity, each independent of the others. The first (dominant) eigenmode defines the field of synchrony. The bottom frames convey the essence of the physical process, which can be explained as follows. The uncorrelated inputs can be decomposed into their even and odd components - roughly, the parts of each driving signal which are coincidentally in phase with the other, and those parts of coincidentally reversed phase - the coincidences 


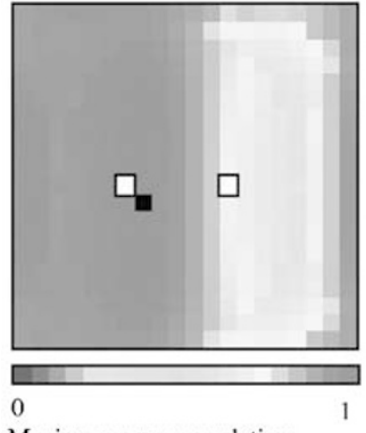

Maximum cross correlation

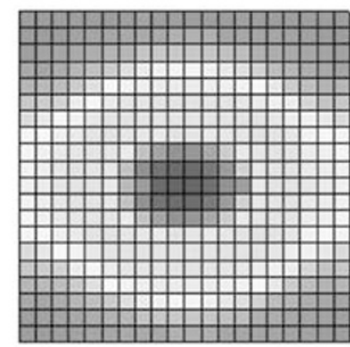

Percent variance: $76.1 \%$

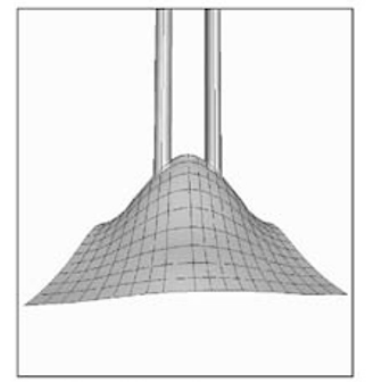

In-phase (even)
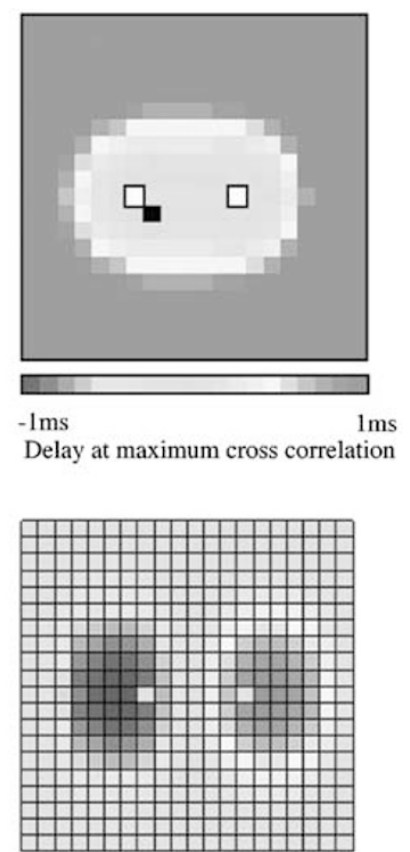

$22.8 \%$

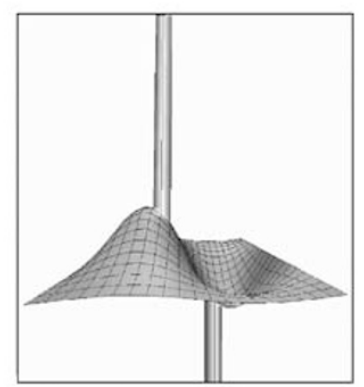

Out-of-phase (odd)

Figure 6 Mechanism of synchrony within the simulated cortex. These figures are derived from a simulated cortical field driven by two uncorrelated white noise inputs, each delivered to one of two sites on the cortical surface. Top: Sites of input shown by white squares. Crosscorrelations and delays, with respect to the reference site at the black square. Middle: first and second eigenmodes of the travelling waves radiating out from the sites of input. Bottom: A schematic representation of the way in which the first and second eigenmodes arise from addition of even components, and cancellation of odd components, in the extended cortical field.

occurring randomly over time. Wave activity radiates from both sites as travelling waves, but since dendrites act as summing junctions, they dissipate even and odd components in their inputs selectively. Even components arriving at each junction reinforce one another, while odd components tend to cancel about the signal mean. Dendrites also perform a running time average of coinciding signals, over a period longer than the axonal conduction lags. Thus, the activity induced by the even components in the driving signals at the two sites dominates throughout the field, and emerges as the first eigenmode of the wave activity.

It may be noted in passing that in this simulation the cortical field remained below transition into the active gamma range, and the boundary conditions of the cortical field were toroidal. Since spatial damping is high, the

boundary conditions are not important. The impact upon synchrony of concurrent transition into autonomous gamma activity at separate cortical locations is currently under investigation.

It can be shown that the magnitude of the zero-lag correlated field is sensitive to dendritic delay time, axonal conduction lag between sites, and the relative strengths of couplings in the field. Figure 7 shows the importance of the strength of coupling between the two active sites. Notably, when two sites on the cortex are concurrently active, the strength of synchronous oscillation between the sites will be a function of both their pre-existing degree of anatomical connectivity, and of the activation of voltage-dependent excitatory receptors. Thus, in these simulations, NMDA acts as a dynamic coupling, enhancing synchrony, and can contribute to the generation of fluctuating fields of synchrony, in appropriate conditions of cortical activation.

\section{INTERACTIONS WITH SUBCORTICAL SYSTEMS}

Figure 8 expands the notions of cortical and subcortical interaction sketched in Figure 1, with emphasis now on the first-order loops of interaction of cortical and thalamic structures. This architecture has been introduced (Robinson et $a l, 2001)$ to a mathematical description of the cortex similar to that described above, but omitting the properties encapsulated in $\Gamma_{p}$ and $s_{q p}$, which confer the capacity for autonomous gamma-band oscillation. Since corticothalamic interactions take place at large scale, between generally quiescent masses of neurones, it has been considered sufficient at present to treat the cortex at large scale as if driven by spatio-temporal white noise, of extrinsic or intrinsic origin. In future, the detailed interaction with locally active cortex must be considered. With that proviso, introduction of coresonance with the thalamus can account for all the additional frequency bands of activity regularly seen in the EEG, in sleep and waking (Walter et al, 1967; Steriade et al, 1990).

Emphasis has been placed on the contribution made to global resonance by the shortest paths of interaction of cortex and subcortex - that is, in loops which are a few neurones long, modulated by interactions between the cortex and the excitatory and inhibitory components within the thalamus, or related systems. Obviously, longer pathways of interaction are of great functional importance, but within the parameters applied in our models, longer pathways cannot generate such relatively 'sharp' resonances, as dendritic processes act as a low-pass filter, progressively blurring the response in loops of many neurones. Multineurone loops appear functionally suited to adiabatic regulation of cortical tone, and thus may be reflected in slow cortical potentials, rather than the major EEG rhythms. Again, this adiabatic regulation is a feature yet to be introduced into any of our models.

Figure 9 shows examples of the way the corticothalamic model can be fitted to EEG power spectra over a wide range of states of cortical activation, from sleep to waking. Here, the process of curve fitting is the inverse of the way that the simulation is used to generate simulated EEG data. In each case, a theoretical curve has been fitted to the experimental data by adjusting a number of free parameters, which are 


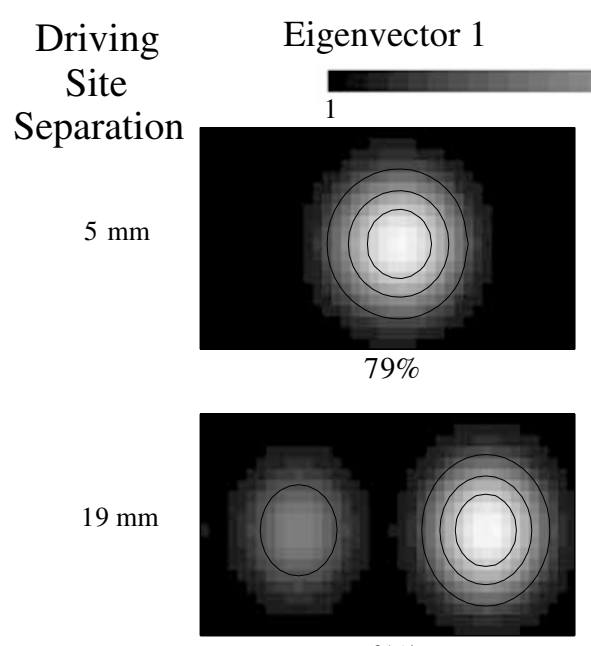

$61 \%$
Eigenvector 2
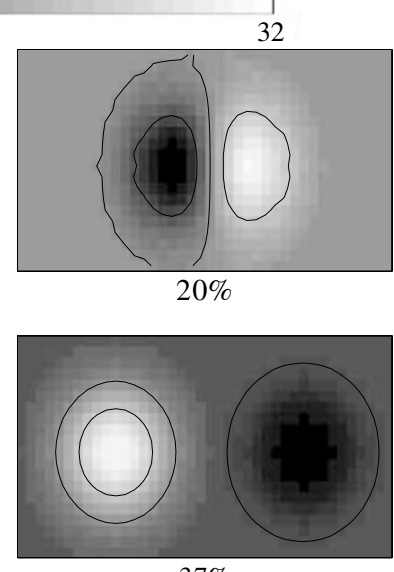

$37 \%$

Gaussian Coupling

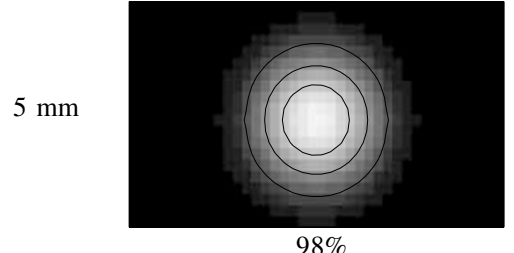

$98 \%$

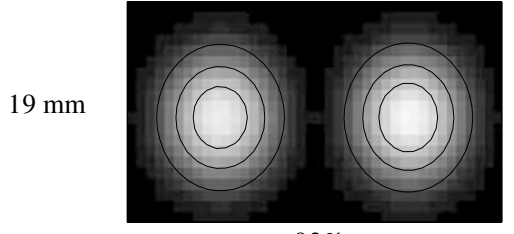

$93 \%$
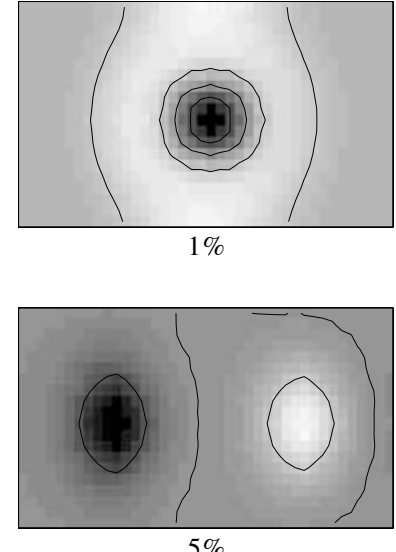

\section{Enhanced Coupling}

Figure 7 Effects of enhanced coupling on synchronous oscillation (compare to middle graphs in Figure 6). First and second eigenmodes of activity for two sites driven by uncorrelated white noise inputs, separated by either 5 or $19 \mathrm{~mm}$. The upper figures show relative proportions of synchronous and antisynchronous (or even and odd) activity when the sites are coupled together only by excitatory surrounds, which decline in strength as a Gaussian function of distance. The lower figures show the impact of enhanced direct coupling between the driven sites, such as would be produced by structured anatomical connections, or by voltage-dependent receptor activation. Top bar shows relative amounts of covariance as shades of gray.

mathematically related to groups of parameters used in the cortical model. As noted above, it is assumed that the system is driven by diffuse white noise, which may be equated with specific and nonspecific inputs to cortex, or with the effect of random perturbations of pulse density partly associated with small patches of autonomous gamma activity, or both. At optimal fit, the free parameters obtained have values in the range expected from the independently estimated parameters used in the cortical model.

A further point of interest is that this account of the origin of the gross EEG spectrum and the major cerebral rhythms does not depend on specific details of the cerebral boundary conditions, as has been proposed by Nunez (1981). In our work it can be readily shown that the spatial damping is very high, and thus global resonant modes play no significant part in the generation of wave activity (Wright, 2000; Robinson et al, 2001). Nunez's (1995) more recent work deals with the interaction of local and global scales of cortical activity, and our present work is in closer harmony with this modification to his earlier work.

Figure 10 extends the explanatory power of the model to include auditory-evoked potentials (ERP). This figure shows real auditory-evoked potentials, obtained by averaging over time-locked epochs of EEG, obtained as the individual was responding to 'target' tones, and ignoring 'nontarget' tones, in a conventional 'oddball' experimental paradigm. Also shown are simulated ERP produced in accord with methods described in Rennie et al (2002). The prestimulus 'resting' EEG of the subject recorded in epochs just before the delivery of each stimulus was characterized by fitting the corticothalamic model in the same way as in Figure 9, to obtain a set of descriptive parameters. These parameters define the transfer function of the corticothalamic system, assuming that the sources driving cortical activity over the prestimulus periods are equivalent to white noise. The 
transfer function thus obtained can then be used to predict the average time-locked response, or impulse response, of the cortex, to any additional, but time-locked, input. As shown, this enables the ERP to be accounted for as timelocked responses to short bursts of activity generated by

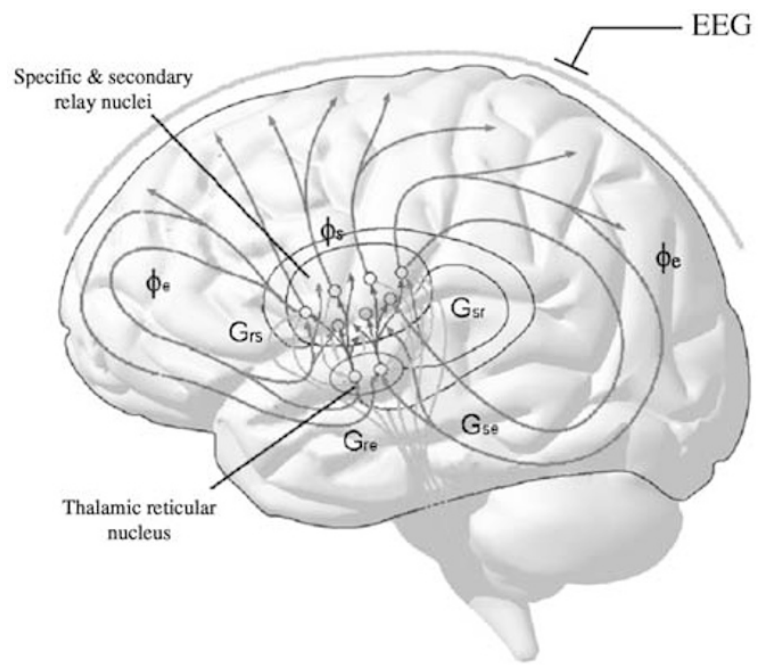

Figure 8 Schematic of interactions between the thalamus and the cortex. The cerebral cortex gives and receives excitatory (red) connections to and from the specific and secondary relay nuclei of the thalamus, and gives connections also to the reticular nucleus of the thalamus. The reticular nucleus gives inhibitory connections to the other thalamic nuclei, and receives inputs from the specific and secondary nuclei. All components are activated by the reticular activating system. Symbols $\phi_{\text {ee, }} \phi_{\text {es }}$, etc, represent pulse fluxes, and $G_{r s}, G_{s r}$ etc, synaptic gains, in accord with a related convention to that used in the purely cortical model. sensory input, and from within the brain. Unfortunately, there is no means currently available to us to generate the second input from within the simulation, as this must depend upon a specific cognitive event-the subjects' decision that the particular tone heard could be (correctly) identified as a target, requiring a response. In principle at least, we expect that this cognitive process might be reduced to associative processes mediated by mechanisms as described in the earlier sections on cortical gamma activity and synchronous oscillation. This step toward closing the paths of causality within brain modelling, is, of course, a daunting task-and one that might best be initially addressed in approximately steady-state conditions, in accord with the methods described by Jirsa et al (2001).

The corticothalamic model so far developed has some interesting implications, which may influence future modelling. Firstly, the role of the lower-frequency cerebral rhythms on biasing transitions of state in the cortex is largely unexplored. Secondly, the occurrence of long-range inhibitory interactions in the thalamus, mediated by the reticular nucleus, contrasts with the predominance of excitation at long range in the cortex. This implies a reversal of phase relations generated by synchronous oscillations in thalamus, to those in cortex. Since thalamic association nuclei and cortex interact with a high degree of one-to-one mapping, the interactions in the entire system might further modulate the onset and offset of fields of synchrony in a dynamic way. Effects on alpha phase suggestive of the operation of such a mechanism have been observed, both experimentally, and in a related type of thalamo-cortical simulation (Suffczynski et al, 2001). Thirdly, the interactions of cortex and thalamus with other subcortical systems over longer pathways may act to modulate the fields of cortical activation, creating analogues
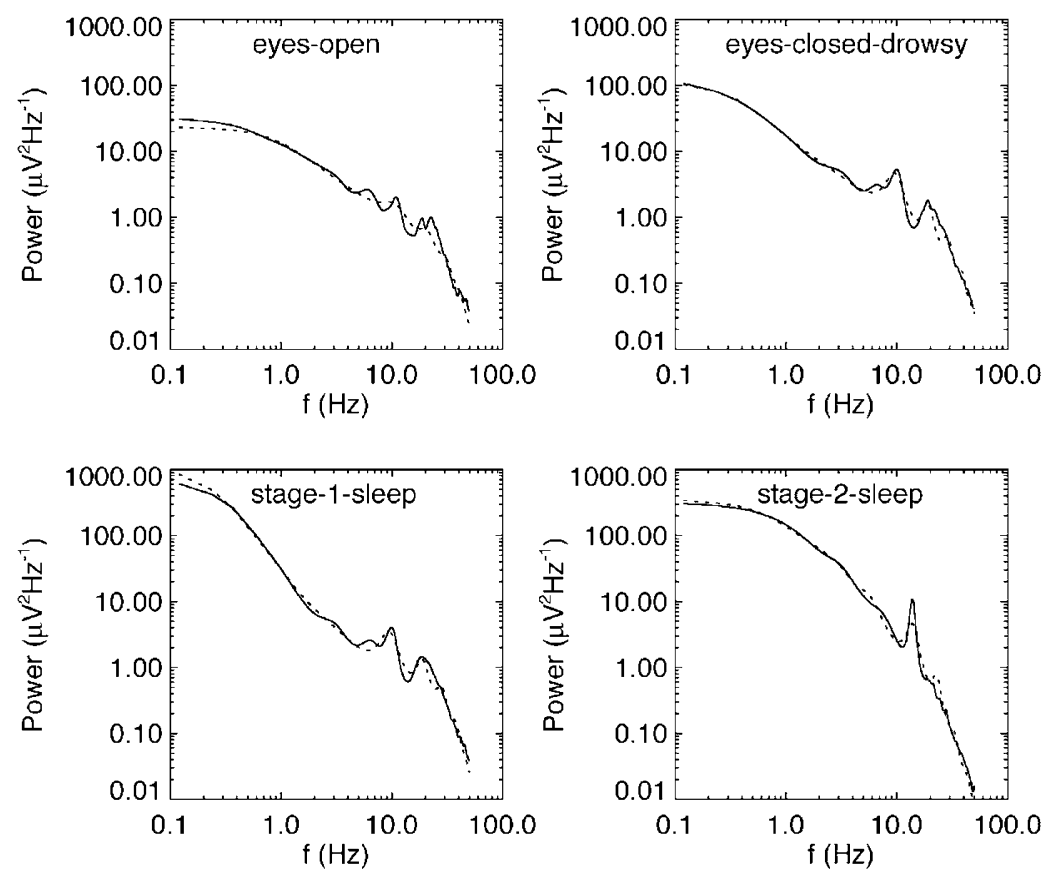

Figure 9 The global EEG and states of cortical activation. Optimum fits of the thalamocortical model to log/log EEG power spectra from the same individual, in alert and drowsy waking states, and the first two stages of sleep. 
a

Model

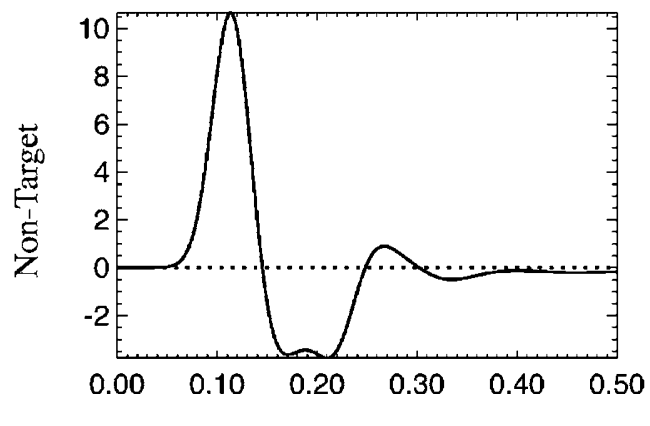

$t(s)$

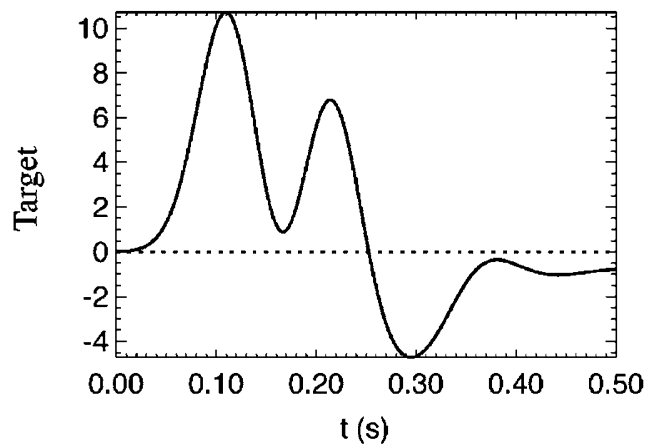

b

Experimental
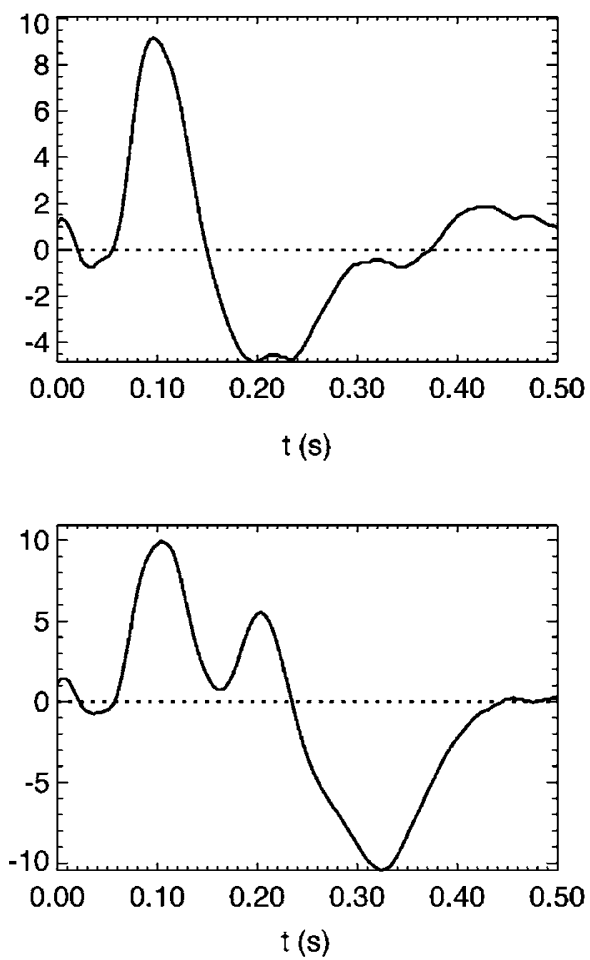

Figure 10 Simulation of the ERP based upon impulse response of the thalamocortical model. Comparison of (a) modelled ERPs with (b) experimental ERPs, in response to target and nontarget tones. To enable modelling of the experimental data, parameter values were obtained by fitting the thalamocortical theoretical model (as in Figure 9) to the EEG power spectrum in the experimental prestimulus epochs. The modelled ERPs were then generated as outputs utilizing the transfer function associated with the prestimulus state, while assuming an input sensory pulse of brief duration reached the subcortex, and hence cortex at $t=0.10 \mathrm{~s}$ from the time of stimulus delivery, for both target and nontarget tones. In the case of target tones, a second impulse was assumed to be generated from autonomous activity within the cortex, a further $0.10 \mathrm{~s}$ later. Thus, the target response is the result of superposition of two impulse responses, generated $0.1 \mathrm{~s}$ apart.

of attention and selective arousal. Such modulations would be relatively slow compared to the cerebral rhythms, as interactions between cortex and thalamus of order greater than two neurones are generally smoothed by dendritic filtering. Subcortical pathways might thus regulate 'adiabatic' shifts in cortical activation, akin to the slow potentials of the cerebral cortex. Fourthly, there is some evidence that some theta activity might be related to pulsed interactions between the cortex and the thalamus (Rennie et al, 2002). Increased understanding of this, and of other sources for theta rhythm may lead to explanation of the failure of our cortical model to account for the theta content observed in experiments, as noted in relation to Figure 3.

Finally, it may be noted that by negative feedback mechanisms, corticothalamic and other cortical/subcortical interactions may contribute to maintaining cortical activation near the level of transition between gamma and $1 / f$ conditions.

\section{DISCUSSION}

The results described above indicate that representation of brain activity in mathematical terms is practicable, albeit in a preliminary manner. Further work is needed to overcome the numerous points upon which our treatment encounters limitations, as pointed out in the preceding text. As such problems are addressed, further physiological and anatomical detail might thus be introduced into an organized framework, always subject to tests against experimentally observed properties of the brain's global properties.

The properties observed in our simulations suggest approximation toward an account of the unified operation of the brain may be possible. Within the simulations there occur properties considered, in related contexts, to confer universal computation, information storage, association and recall, and self-organization (Freeman and Rogers, 2002; Arhem and Liljenstrom, 2001; Liljenstrom, 2002; Langton, 1986, 1990; Singer, 1994; von Neumann, 1949; Wolfram, 1984; Phillips and Singer, 1997; Kording and Konig, 2000). Work showing that realistic anatomical connectivity can appear on the basis of Hebbian learning, in certain simplified neural networks (von der Marlsburg, 1973; Swindale, 1996; Alexander et al, 2000) is also of interest, as this property appears likely to be transferable to the more dynamically realistic continuum models described here.

A further step would see, as well as the introduction of Hebbian learning, the incorporation of innate positive and negative reinforcement systems analogous to those known to exist in the brain (Olds and Milner, 1954). It may be presumed that such reinforcement systems act, via neuro- 
modulation, to regulate synaptic consolidation. Some survival behaviors related to activity in the reinforcement pathways appear to be hard-wired, thanks to species evolution. Such a priori reinforcement systems might also act to supervise adaptive learning, if the reinforcement system was initially activated by only a small subset of the total environmental stimuli, but could, by associative learning, come to be activated by more complex inputs, and by internally generated brain states. Internally generated states that did not acquire association with basic survival behaviors and the operation of the reinforcement mechanisms would be extinguished if they did not activate the survival behaviors and the synaptic consolidation mechanisms over some critical time period. Thus, survival-consistent behaviors would remain, and develop in complexity.

Another goal is the fitting of models of this type to a much larger range of data, particularly the large normative and standardized data set under construction by Gordon (2000, 2002), which includes EEG and fMRI data from both normal people and people with a variety of psychopathologies. It is our hope that this will help us to contribute to the unification of findings in neuropsychopharmacology, as discussed at the beginning of this paper.

\section{ACKNOWLEDGEMENTS}

We thank Professor Walter Freeman for access to his library of electrocortical recordings, Mr Nicholas Hawthorn for technical assistance, and the University of Sydney, the University of Auckland, the Swinburne University, Melbourne, and the Mental Health Research Institute, Melbourne, for the use of computer facilities. We thank also our hosts at the Agora for BioSystems, Stockholm, and the University of Potsdam, at whose conferences this paper was delivered.

\section{REFERENCES}

Alexander DM, Bourke PD, Sheridan P, Konstandatos O, Wright JJ (2000). Emergence under Hebbian learning of local maps in the primary visual cortex: orientation preference in the tree shrew. Unpublished manuscript, available at http://www.mhri.edu.au/ bdl/.

Alexander GE, Crutcher MD, DeLong MR (1990). Basal gangliathalamocortical circuits: parallel substrates for motor, oculomotor, 'prefrontal' and 'limbic' functions. In: Uylings HBM, van Eden CG, de Bruin JPC, Feenstra MGP (eds). Progress in Brain Research. Elsevier: Amsterdam.

Amit DJ (1989). Modelling Brain Function. The World of Attractor Neural Networks. Cambridge University Press: New York.

Arbib MA, Erdi P, Szentagothai J (1998). Neural Organisation: Structure, Function and Dynamics. MIT Press: Cambridge, MA London.

Arhem P, Liljenstrom H (2001). Fluctuations in neural systems: from subcellular to network levels. In: Moss F, Gielen S (eds). Handbook of Biological Physics. Vol. 4: Neuro-Informatics, Neural Modelling. Elsevier: Amsterdam. pp 83-125.

Basar E (1976). Biological and Physiological Systems Analysis. Addison-Wesley: Reading, MA.

Braitenberg V, Schuz A (1991). Anatomy of the Cortex: Statistics and Geometry. Springer: Berlin.
Bressler SL, Coppola R, Nakamura R (1993). Episodic multiregional cortical coherence at multiple frequencies during visual task performance. Nature 366: 153-156.

Chapman CL, Bourke PD, Wright JJ (2002). Spatial eigenmodes and synchronous oscillation: coincidence detection in simulated cerebral cortex. J Math Biol 45: 57-78.

Dominguez-Perrot C, Feltz P, Poulter MO (1996). Recombinant GABAa receptor desensitization: the role of the gamma2 subunit and its physiological significance. J Physiol 497: 145-159.

Eckhorn R, Bauer B, Jordon W, Brosch M, Kruse W, Munk M et al (1988). Coherent oscillation: a mechanism of feature linking in visual cortex? Biol Cybern 60: 121-130.

Freeman WJ (1975). Mass Action in the Nervous System. Academic Press: New York.

Freeman WJ, Barrie JM (2000). Analysis of spatial patterns of phase in neocortical gamma EEGs in rabbit. J Neurophysiol 84: $1266-1278$.

Freeman WJ, Rogers LJ (2002). Fine temporal resolution of analytic phase reveals episodic synchronisation by state transitions in gamma EEGs. J Neurophysiol 87: 937-945.

Gordon E (2000). In: E Gordon (ed). Integrative Neuroscience. Harwood Academic: Australia.

Gordon E (2002). Integrative neuroscience in psychiatry. Paper read at the Abisco Conference, Agora for Biosystems, Royal Swedish Academy of Science.

Gray CM, Konig P, Engel AK, Singer W (1989). Oscillatory responses in cat visual cortex exhibit intercolumnar synchronisation which reflects global stimulus properties. Nature 388: 334-337.

Gray CM, Singer W (1989). Stimulus-specific neuronal oscillations in orientation columns of cat visual cortex. Proc Natl Acad Sci 86: $1698-1702$.

Haken H, Kelso JAS, Bunz H (1985). A theoretical model of phase transitions in human hand movements. Biol Cybern 51: 347-356.

Hausser W, Roth A (1997). Dendritic and somatic glutamate receptor channels in rat cerebellar Purkinje cells. J Physiol 501.1: 77-95.

Jirsa VK, Haken H (1996). Field theory of electromagnetic brain activity. Phys Rev Lett 77: 960-963.

Jirsa VK, Jantzen KJ, Fuchs A, Kelso JAS (2001). Neural field dynamics on the folded three-dimensional cortical sheet, and its forward EEG and MEG. In: Insana MF, Leahy RM (eds). IPMI 2001, Lecture Notes in Computer Science, Vol. 2082. Springer: Berlin, Heidelberg. pp 286-299.

Kay J, Phillips WA (1997). Activation functions, computational goals and learning rules for local processors with contextual guidance. Neural Comput 9: 763-768.

Kording KP, Konig P (2000). A learning rule for dynamic recruitment and decorrelation. Neural Networks 13: 1-9.

Langton CD (1986). Studying artificial life with cellular automata. Physica D 22: 120-149.

Langton CD (1990). Computation at the edge of chaos: phase transitions and emergent computation. Physica D 42: 12-37.

Lester RA, Jahr CE (1992). NDMA channel behavior depends on agonist affinity. $J$ Neurosc 12: 635-643.

Liley DTJ, Cadusch PJ, Daflis MP (2002). A spatially continuous mean field theory of electrocortical activity. Network: Comput Neural Systems 13: 67-113.

Liley DTJ, Cadusch PA, Wright JJ (1999). Continuum theory of electro-cortical activity. Neurocomputing 26-27: 795.

Liley DTJ, Wright JJ (1994). Intracortical connectivity of pyramidal and stellate cells: estimates of synaptic densities and coupling symmetry. Network 5: 175-189.

Liljenstrom, H (2002). Regulating cortical neurodynamics for past, present and future 5th international conference on computing anticipatory systems 2001 (CASYS'01). In: Dubois DM (ed). AIP Conference Proceedings. American Institute of Physics: New York. 
Livingstone MS (1996). Oscillatory firing and interneuronal correlations in squirrel monkey striate cortex. J Neurophysiol 75: 2467-2485.

McCulloch WS, Pitts W (1943). A logical calculus of the ideas immanent in nervous activity. Bull Math Biophys 5: 115-133.

von der Marlsburg C (1973). Self-organization of orientation selective cells in the striate cortex. Kybernetic 14: 85-100.

Miltner WH, Braun C, Arnold M, Witte H, Taube E (1999). Coherence of gamma-band EEG activity as a basis for associative learning. Nature 397: 434-436.

Neuenschwander S, Singer W (1996). Long range synchronisation of oscillatory light responses in the cat retina and lateral geniculate nucleus. Nature 379: 728-733.

von Neumann J (1949). Theory of self-reproducing automata. In: Burkes AW (ed). University of Illinois Lectures on the Theory and Organisation of Complicated Automata. University of Illinois Press: Urbana, IL.

Nunez PL (1981). Electric Fields of the Brain. Oxford University Press: New York.

Nunez PL (1995). Neocortical Dynamics and Human EEG Rhythms. Oxford University Press: New York.

Olds J, Milner P (1954). Positive reinforcement produced by electrical stimulation of the septal area and other regions of the rat brain. J Comp Physiol Psychol 47: 419-427.

Palm G, Wennekers T (1997). Synchronicity and its use in the brain. Behav Brain Sci 20: 695-696.

Partin KM, Fleck MW, Mayer ML (1996). AMPA receptor flip/flop mutants affecting deactivation, desensitization and modulation by cyclothiazide, aniracetam and thiocyanate. J Neurosci 16: 6634-6647.

Phillips WA, Singer W (1997). In search of common foundations for cortical computation. Behav Brain Sci 20: 657-722.

Phillips WA, Pflieger ME (1999). EEG studies of interactions that coordinate cortical activity. In: Nakada $\mathrm{T}$ (ed). Human Higher Function: I Advanced methodologies. Smith-Gordon/ Nishuma.

Posner MI, Petersen SE (1990). The attention system of the human brain. Annu Rev Neurosci 13: 25-42.

Rennie CJ, Robinson PA, Wright JJ (1999). Effects of local feedback on dispersion of electrical waves in the cerebral cortex. Phys Rev E 59: 3320-3329.

Rennie CJ, Robinson PA, Wright JJ (2002). Unified neurophysical model of EEG spectra and evoked potentials. Biol Cybernet 86: 457-471.

Rennie CJ, Wright JJ, Robinson PA (2000). Mechanisms of cortical electrical activity and the emergence of gamma rhythm. $J$ Theoret Biol 205: 17-35.

Robinson PA, Loxley PN, O'Connor SC, Rennie CJ (2001). Modal analysis of corticothalamic dynamics, electroencephalographic spectra, and evoked potentials. Phys Rev E 63: 041909.

Robinson PA, Rennie CJ, Wright JJ (1997). Propagation and stability of waves of electrical activity in the cerebral cortex. Phys Rev E 56: 826-840.

Robinson PA, Rennie CJ, Wright JJ, Bahramali H, Gordon E, Rowe DL (2001). Prediction of electroencephalographic spectra from neurophysiology. Phys Rev E 63: 021903.

Robinson PA, Rennie CJ, Wright JJ, Bourke PD (1998b). Steady states and global dynamics of electrical activity in the cerebral cortex. Phys Rev E 58: 3557-3571.

Robinson PA, Wright JJ, Rennie CJ (1998a). Synchronous oscillations in the cerebral cortex. Phys Rev E 57: 4578-4588.

van Rotterdam A, Lopes da Silva FH, van den Ende J, Viergever MA, Hermans AJ (1982). A model of the spatio-temporal characteristics of the alpha rhythm. Bull Math Biol 44: 283-305.

Singer W (1994). Putative functions of temporal correlations in neocortical processing. In: Koch C, Davis JL (eds). Large Scale
Neuronal Theories of the Brain. MIT Press: Cambridge, MA, London.

Singer W, Gray CM (1995). Visual feature integration and the temporal correlation hypothesis. Annu Rev Neurosci 18: 555-586.

Steriade M, Amzica F, Contreras D (1996). Synchronisation of fast $(30-40 \mathrm{~Hz})$ spontaneous cortical rhythms during brain activation. J Neurosci 16: 392-417.

Steriade M, Gloor P, Llinas RR, Lopes da Silva FH, Mesulam MM (1990). Basic mechanisms of cerebral rhythmic activities. Electroencephalogr Clin Neurophysiol 76: 481-508.

Steriade M, Timofeev I, Grenier F (2001). Natural waking and sleep states: a view from inside cortical neurons. J Neurophysiol 85: 1969-1985.

Stryker MP (1989). Is grandmother an oscillation? Nature 388: 297-298.

Stuart GJ, Sakmann B (1994). Active propagation of somatic action potentials into neocortical cell pyramidal dendrites. Nature 367: 69-72.

Suffczynski P, Kalitzin S, Pfurtscheller G, Lopes da Silva FH (2001). Computational model of thalamocortical networks: dynamical control of alpha rhythms in relation to focal attention. Int $J$ Psychophysiol 43: 25-40.

Swindale NV (1996). The development of topography in the visual cortex: a review of models. Network 7: 161-247.

Thomson AM (1997). Activity dependent properties of synaptic transmission at two classes of connections made by rat neocortical pyramidal neurons in vitro. J Physiol 502: 131-147.

Thomson AM, West DC, Hahn J, Deuchars J (1996). Single axon IPSP's elicited in pyramidal cells by three classes of interneurones in slices of rat neocortex. J Physiol 496: 81-102.

Tones MV, Westbrook GL (1996). The impact of receptor desensitization on fast synaptic transmission. Trends Neurosci 19: 96-101.

Traub RD, Whittington MA, Stanford IM, Jeffereys JGR (1996). A mechanism for generation of long range synchronous fast oscillations in the cortex. Nature 383: 621-624.

Walter DO, Kado BS, Rhodes JM, Adey WR (1967). Electroencephalographic baselines in astronaut candidates estimated by computation and pattern recognition techniques. Aerosp Med 38: 371-379.

Wilson HR, Cowan JD (1973). A mathematical theory of the functional dynamics of cortical and thalamic nervous tissue. Kybernetik 13: 55-80.

Wolfram S (1984). Universality and complexity in cellular automata. Physica D 10: 1-35.

Wright JJ (1990). Reticular activation and the dynamics of neuronal networks. Biol Cybernet 62: 289-298.

Wright JJ (1997a). EEG simulation: variation of spectral envelope, pulse synchrony and approx. $40 \mathrm{~Hz}$ oscillation. Biol Cybernet 76: 181-194.

Wright JJ (1997b). Local attractor dynamics will introduce further information to synchronous neuronal fields. Behav Brain Sci 20: 701-702.

Wright JJ (1999). Simulation of EEG: dynamic changes in synaptic efficacy, cerebral rhythms, and dissipative and generative activity in cortex. Biol Cybernet 81: 131-147.

Wright JJ (2000). Developing testable theories of brain dynamics: the global mode theory and experimental falsification. Behav Brain Sci 23: 414-415.

Wright JJ, Bourke PD, Chapman CL (2000). Synchronous oscillation in the cerebral cortex and object coherence: simulation of basic electrophysiological findings. Biol Cybernet 83: 341-353.

Wright JJ, Liley DTJ (1996). Dynamics of the brain at global and microscopic scales. Neural networks and the EEG. Behav Brain Sci 19: 285-320. 
APPENDIX: A MORE DETAILED DESCRIPTION OF THE MATHEMATICS OF A CORTICAL MODEL AND OF METHODS OF SIMULATION

In the following formulae, subscript $p$ represents association with a presynaptic neurone, and subscript $q$ association with a postsynaptic neurone. Thus $p$ or $q$ may each be replaced by either $e$ (excitatory) or $i$ (inhibitory), and subscript $q p$ indicates a shared property. The extended subscripts $i c$ and $c c$ indicate that either intracortical or corticocortical synapses are specifically referred to.

The principal state variables are:

$Q_{q}(\mathbf{r}, t)$, the point pulse densities of neurones of type $q$, where $t$ is the time, and $\mathbf{r}$ is the position on the cortical surface $\left(\mathrm{s}^{-1}\right)$.

$V_{q}(\mathbf{r}, t)$, the corresponding point local field potentials (V).

$\varphi_{p}(\mathbf{r}, t)$, the synaptic flux densities $\left(\mathrm{s}^{-1}\right)$.

The rate at which pulses are generated is given by

$$
Q_{q}=Q_{q}^{\max } / 1+\mathrm{e}^{-\pi\left(V_{q}-\theta_{q}\right) / \sqrt{3} \sigma_{q}},
$$

where $Q_{q}{ }^{\max }$ is the maximum pulse rate sustainable by neurones of type $q$, and $\theta_{q}$ and $\sigma_{q}$ are parameters defining pulse density distribution as a function of $V_{q}$. Perturbation of the value of $Q_{q}(\mathbf{r}, t)$ by random local factors in individual neurones provides an internal source of white noise driving to the system.

The synaptic flux densities are given by equations of the form

$$
\varphi_{p}=\int f\left(\left|\mathbf{r}-\mathbf{r}^{\prime}\right|, \mathbf{r}_{p}\right) Q_{p}\left(\mathbf{r}^{\prime}, t-\left|\mathbf{r}-\mathbf{r}^{\prime}\right| / v_{p}\right) \mathrm{d}^{3} \mathbf{r}^{\prime},
$$

where $\mathbf{r}_{p}$ is the axonal range and $v_{p}$ is the average velocity of axonal conduction. The axonal distribution functions $f\left(\left|\mathbf{r}-\mathbf{r}^{\prime}\right|, \mathbf{r}_{p}\right)$ may have any form, but are usually treated as two-dimensional Gaussian distributions. Intracortical connections are also usually approximated as having no significant extension in space, unlike the corticocortical connections, and thus

$$
\begin{aligned}
& \varphi_{e}=\varphi_{e, i c}+\varphi_{e, c c}, \\
& \varphi_{e, i c} \approx Q_{e}, \\
& \varphi_{i, i c} \approx Q_{i},
\end{aligned}
$$

$\varphi_{s}$ and $\varphi_{n s}$ are specific and nonspecific inputs to the cortex from all sources, and are not explicitly described here, but act as an external source of driving inputs to the cortical system.

Local field potential is considered directly proportional to the pyramidal cell point membrane potential, which is given by

$$
V_{q}=V_{q}^{[0]}+\sum_{p} N_{q p} H_{q p}^{[D]} \otimes\left(s_{q p} \Gamma_{p} \varphi_{p}\right),
$$

where $V_{q}^{[0]}$ is the resting membrane potential, $N_{q p}$ is the number of synapses of type $p$ per dendritic tree, and $\otimes$ indicates convolution over time.
Normalized average EPSP/IPSP at the soma are given by

$$
H_{q p}^{[D]}(t)=\frac{a_{q p} b_{q p}}{b_{q p}-a_{q p}}\left(\mathrm{e}^{-a_{q p} t}-\mathrm{e}^{-b_{q p} t}\right), \quad t \geqslant 0,
$$

where $1 / b_{q p}, 1 / a_{q p}$ are the rise and fall time constants.

The standardized synaptic strength is represented by $g_{p}^{[0]}$. It is the integral over time of the PSP, as recorded at the soma, when the neurone is at its resting membrane potential $V_{q}^{[0]}$. The actual synaptic strength $s_{q p}$ is related to this, through the ion channel reversal potentials, $V_{q, p}^{\mathrm{rev}}$, and a measure of the membrane potential, thus:

$$
s_{q p}=g_{p}^{[0]} \frac{V_{p}^{\mathrm{rev}}-H_{q}^{[M]} \otimes V_{q}}{V_{p}^{\mathrm{rev}}-V_{q}^{[0]}} .
$$

The convolution by $H_{\mathrm{q}}^{[M]}$ is introduced to account for an effect of retrograde propagation of action potentials into the dendritic tree. We assume that at low synaptic flux and firing rates, cells are principally sensitive to synapses proximate to the soma. During active firing, the site of synaptic summation and further action potential generation is shifted more distally, into the dendritic tree, and now more remote synapses contribute greater weight to determination of whether or not firing continues. The contribution of remote synapses depends upon the arrival of EPSP, IPSP generated at delay times in the distal dendrites, and at earlier values of the membrane potential. For the population of cells, the delay because of this effect increases as average membrane potential decreases, so to first approximation,

$$
H_{q}^{[M]}=c_{q} \mathrm{e}^{-c_{q} t}, \quad t \geqslant 0,
$$

where $c_{q}$ is determined by a linear regression,

$$
c_{q}=a\left(V_{q}-V_{q}^{[0]}\right)+b .
$$

(The linear regression can be replaced by a more physiologically realistic nonlinear function, which will be reported in detail elsewhere.)

The impact of this membrane-voltage-dependent switching of a mean delay process in the dendrites is to regulate partially the transition of local electrocortical activity into, and out of, an active state in which gamma activity appears, and generates strong fluctuations in the local pulse-density and local field potential. This contributes a third type of driving to wave propagation in the system, in addition to that provided from external sources and stochastic perturbations of pulse density.

Neurotransmitter receptor adaptation to continuing input is included in $\Gamma_{p}$, for which parameters $\kappa_{p}^{[R]}, \lambda[R], A_{n}, \alpha_{n}, B_{n}, \beta_{n}$ have been obtained from modelling the behavior of receptors, from physiological measurements of transformations among receptor tertiary molecular configurations (references are given in main text).

$$
\Gamma_{p}=H_{p}^{\left[R_{c}\right]} \otimes \Gamma_{p}^{\left[R_{c}\right]}+H_{p}^{\left[R_{V}\right]} \otimes \Gamma_{p}^{\left[R_{V}\right]} .
$$

The superscript $[R]=\left[R_{c}\right],\left[R_{V}\right]$ indicates whether or not the receptor is voltage dependent. Thus if $p=e, R_{c}$ indicates characteristics of an AMPA receptor, and $R_{V}$ an NMDA receptor, while if $p=i$, then $R_{c}$ indicates a GABAa receptor, 
and no voltage-dependent channels are considered.

$$
\begin{aligned}
\Gamma_{p}^{\left[R_{c}\right]} & =\kappa_{p}^{\left[R_{c}\right]} \mathrm{e}^{-\lambda\left[R_{c}\right] \phi_{p}}, \\
\Gamma_{p}^{\left[R_{v}\right]} & =\kappa_{p}^{\left[R_{V}\right]} \mathrm{e}^{-\lambda\left[R_{V}\right] \phi_{p}} \times Q_{q} / Q_{q}^{\max } .
\end{aligned}
$$

The coefficients $\kappa_{p}^{[R]}$ describe the relative amplitude of the two components of $\Gamma_{p}$, and the multiplication by $Q_{q} / Q_{q}{ }^{\max }$ is introduced in the second of the above equations because voltage-dependent receptors are active only in that fraction of neurones, which are currently firing.

The onset and offset of this modulation of synaptic gain are described by normalized time functions, analogous to $H_{q p}^{[D]}$ :

$$
\begin{aligned}
& H_{p}^{[R]}(t)=\left[B_{1}^{[R]} / \beta_{1}^{[R]}+B_{2}^{[R]} / \beta_{2}^{[R]}+\cdots-A_{1}^{[R]} / \alpha_{1}^{[R]}\right. \\
& \left.-A_{2}^{[R]} / \alpha_{2}^{[R]}-\cdots\right]^{-1} \times\left[B_{1}^{[R]} \mathrm{e}^{-b_{1}^{[R]_{t}}}\right.
\end{aligned}
$$

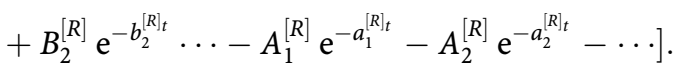

These modulations of synaptic gain help to maintain cortical stability close to the boundary between $1 / f$ activity, and active gamma, since AMPA activity is more strongly downregulated with decreasing membrane voltage than is GABA activity. The voltage dependence of NMDA activity contributes a type of dynamic gain, enhancing synchronous oscillation.

The simulation results shown in Figures 3 and 4 applied random perturbations of the pulse densities $Q_{q}(\mathbf{r}, t)$ in accord with the assumed stochastic nature of individual neurone firing. In Figures 5, 6, and 7, this perturbation was not applied, although the moving bars or static inputs were treated as zero-mean signals of white-noise type. The theoretical functions applied to derive the results in Figures 9 and 10 assumed driving of the model system by diffuse white noise. These differences represent, in part, stages in the evolution of the family of models rather than differences of principle. All relevant properties are retained in simulations that include the more complex treatments of the sources of driving.

The cortical simulations were applied with the cortical surface 'lumped' into a $20 \times 20$ matrix of elements, with toroidal boundary conditions. Variation of boundary conditions is without significant effect. 